\title{
Pseudomicroangiopathie thrombotique par carence en vitamine B12
}

\section{Pseudothrombotic Microangiopathy Related to Vitamin B12 Deficiency}

\author{
X. Dubucs $\cdot$ D. Lauque $\cdot$ L. Astudillo \\ Reçu le 20 octobre 2017; accepté le 31 janvier 2018 \\ (C) SFMU et Lavoisier SAS 2018
}

\section{Introduction}

L'anémie aiguë est un motif fréquent de recours aux urgences. Outre l'origine hémorragique, il existe des diagnostics à ne pas méconnaître qui constituent d'authentiques urgences hématologiques. L'analyse attentive des signes clinicobiologiques en suivant une démarche étiologique progressive permet une orientation certaine vers le mécanisme sous-jacent.

\section{Observation}

Un patient de 76 ans est adressé par son médecin traitant aux urgences pour une suspicion d'anémie hémolytique. Il a pour seul antécédent une hypertension artérielle ancienne, traitée par amlodipine. Le patient rapporte une asthénie depuis un mois et depuis quelques jours une dyspnée d'effort qui conduit le patient à consulter son médecin traitant. Ce dernier lui prescrit un bilan biologique révélant l'anémie. Son taux d'hémoglobine était mesuré à 13,4 g/dl lors d'un bilan systématique six mois auparavant.

L'examen clinique à son arrivée aux urgences est rassurant, avec une tension artérielle à 120/80 $\mathrm{mmHg}$, une fréquence cardiaque à $80 / \mathrm{min}$, une fréquence respiratoire à $17 /$ min avec une saturation à $98 \%$ en air ambiant et un indice de masse corporelle calculé à $33,9 \mathrm{~kg} / \mathrm{m}^{2}(176 \mathrm{~cm}$, $105 \mathrm{~kg}$ ). Il existe une pâleur cutanéomuqueuse associée à un discret ictère conjonctival, l'examen abdominal est sans particularité. Il n'existe pas de symptomatologie hémorragique extériorisée. L'examen cardiopulmonaire est rassu-

\footnotetext{
X. Dubucs $(\bowtie) \cdot$ D. Lauque $\cdot$ L. Astudillo

CHU de Toulouse, bâtiment urgences réanimation médecine, place du Docteur-Baylac, F-31300 Toulouse, France

e-mail : xavier.dubucs@gmail.com

D. Lauque

Université Toulouse-III, Paul-Sabatier,

118, route de Narbonne, F-31062 Toulouse, France
}

rant, sans signe d'insuffisance cardiaque. L'examen neurologique est normal. L'ECG s'inscrit en rythme sinusal avec un bloc de branche droit complet. Le bilan biologique est contrôlé et complété dès son arrivée : hémoglobine $6,5 \mathrm{~g} / \mathrm{dl}$ avec un VGM augmenté à $121 \mu^{3}$, plaquettes $135000 /$ $\mathrm{mm}^{3}$, leucocytes à $4,750 \mathrm{~g} / 1$ avec une formule leucocytaire normale. Les réticulocytes sont bas à $36900 / \mathrm{mm}^{3}$, et il est mis en évidence de rares schizocytes. Il existe des stigmates d'hémolyse avec une haptoglobine effondrée $(<0,1 \mathrm{~g} / \mathrm{l})$, des LDH augmentés à $2119 \mathrm{UI} / 1$ (normale $<210 \mathrm{UI} / 1)$ et une augmentation de la bilirubine libre mesurée à $44,4 \mu \mathrm{mol} / 1$. Le test de Coombs revient négatif. Le reste du bilan est normal (bilan hépatique, fonction rénale). Du fait de la bonne tolérance clinique, le patient n'est pas transfusé et est transféré en service de médecine interne. Le résultat des dosages vitaminiques, disponible le lendemain, met en évidence une carence profonde en vitamine B12 inférieure à $100 \mathrm{pg} / \mathrm{ml}$ (normale $>615 \mathrm{pg} / \mathrm{ml}$ ). Les dosages plasmatiques des anticorps antifacteurs intrinsèques et anticellules pariétales de l'estomac sont négatifs. Le patient bénéficie d'une supplémentation intramusculaire de vitamine B12, $1 \mathrm{mg} / \mathrm{j}$ pendant dix jours constituant le traitement d'attaque relayé par une injection une fois par mois. Le taux d'hémoglobine à cinq jours remontant à $9,1 \mathrm{~g} / \mathrm{dl}$ permet le retour à domicile. À deux mois du début de la supplémentation, l'hémoglobine est à 12,6 g/dl, la fibroscopie digestive haute réalisée à ce moment retrouve une atrophie gastrique caractéristique de la maladie de Biermer. À noter que la recherche d'infection à Helicobacter pylori est négative.

\section{Discussion}

Le diagnostic posé est donc une pseudomicroangiopathie thrombotique résultant d'une carence en vitamine B12 qui mime, comme son nom l'indique, une véritable microangiopathie thrombotique (MAT).

L'association d'une anémie à une thrombopénie avec présence de schizocytes fait craindre en premier lieu une MAT [1]. Cette entité se décline en deux catégories : le syndrome 
hémolytique et urémique et le purpura thrombotique thrombocytopénique. Le premier présente un tropisme rénal, avec un risque d'insuffisance rénale aiguë, tandis que le second atteint plutôt le système nerveux central, pouvant conduire notamment à un état de mal épileptique. Le pronostic vital peut être rapidement engagé par pluridéfaillance d'organes. La MAT est donc une urgence thérapeutique qui nécessite une prise en charge spécialisée au sein d'un centre de référence proche d'un service de soins intensifs, afin d'envisager des échanges plasmatiques répétés et une thérapeutique immunosuppressive.

La distinction entre MAT et pseudo-MAT est primordiale afin d'éviter des errances diagnostiques et des mesures thérapeutiques inadaptées potentiellement invasives [2].

Le dosage des réticulocytes est la pierre angulaire de la démarche diagnostique devant une anémie normo/macrocytaire isolée ou une bicytopénie, afin de déterminer son caractère régénératif. En cas de MAT, l'anémie est régénérative, avec des réticulocytes élevés, conséquence d'une destruction mécanique des hématies secondaires à une cascade d'activation inappropriée de la coagulation et d'activation plaquettaire. La vigilance est de mise dans cette pathologie, car les schizocytes qui sont des hématies fragmentées circulantes peuvent apparaître de façon retardée, nécessitant leur recherche répétée dans les 72 premières heures [3]. Dans la série de Noël et al., les caractéristiques de sept patients présentant une pseudo-MAT ont été comparées avec celles de six patients atteints d'une MAT [4]. L'âge moyen des patients présentant une pseudo-MAT était de 72 ans $(p=0,05)$ contre 35 ans dans le groupe MAT. Le taux de réticulocyte était significativement plus bas dans le premier groupe $\left(13,1\right.$ vs $\left.265,5 \times 10^{9} / 1 ; p=0,0012\right)$ et le taux plaquettaire plus élevé $\left(73,0\right.$ vs $12,5 \times 10^{9} / 1$; $p=0,0023)$. L'anémie était macrocytaire en cas de pseudo-MAT $\left(110 \mu^{3}\right.$ [96,3-130]) et plus souvent normocytaire en cas de MAT $\left(92 \mu \mathrm{m}^{3}[82-118] ; p=0,05\right)$.

Rappelons que la carence en vitamine B12 se manifeste le plus souvent par une anémie macrocytaire isolée, voire une pancytopénie. Plus rarement, en cas de carence profonde en vitamine B12, les hématies fortement augmentées de volume perdent leur capacité à se déformer dans les capillaires et peuvent être hémolysées [5]. Il existe également une hémolyse intramédullaire du fait d'une érythropoïse inefficace [6]. Ces mécanismes expliquent les stigmates d'hémolyse (subictère, LDH augmentée, bilirubine libre augmentée, haptoglobine effondrée) qui peuvent orienter à tort vers une anémie d'origine périphérique. Les schizocytes mis en évidence témoignent du processus mécanique de l'hémolyse.

La maladie de Biermer est une maladie auto-immune caractérisée par une destruction de la muqueuse gastrique, conduisant à la réduction de la synthèse du facteur intrinsèque qui se lie normalement à la vitamine $\mathrm{B} 12$ afin de per-

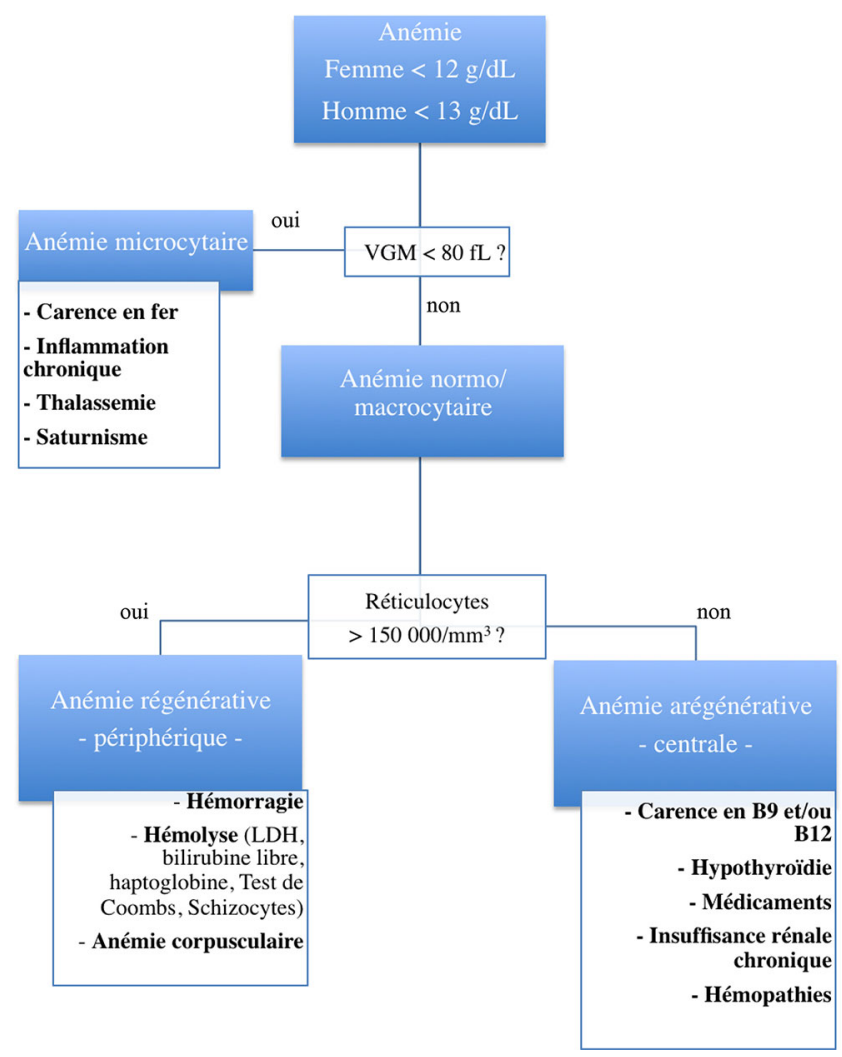

Fig. 1 Orientation diagnostique devant une anémie aux urgences dl : décilitre ; fl : femtolitre ; LDH : lactase déshydrogénase ; VGM : volume globulaire moyen

mettre son absorption au niveau de l'iléon distal. Elle représente $20 \%$ des étiologies de carence en vitamine B12 [7]. L'âge moyen au diagnostic est de 74 ans avec un sex-ratio H/ $\mathrm{F}$ de 2,9 [8]. Le traitement consiste en une supplémentation à vie par vitamine B12, par voie intramusculaire ou per os, avec initialement un traitement d'attaque par une ampoule de vitamine B12 par jour pendant 10 jours, puis un traitement d'entretien par une ampoule par mois [9].

En dehors des carences d'apport, les autres étiologies de carences en vitamine B12 à ne pas méconnaître sont les gastrectomies partielles ou totales, les pathologies de l'iléon terminal (maladie de Crohn...), ou plus fréquemment le syndrome de non-dissociation de la vitamine B12 et de ses protéines porteuses, pouvant être favorisé par la prise d'inhibiteur de la pompe à protons [10].

En conclusion, ce cas clinique illustre l'exigence de l'approche étiologique de l'anémie et des bicytopénies. La lecture systématique de l'anémie en suivant l'algorithme étiologique rappelé ci-dessous (Fig. 1) permet une orientation juste sur le mécanisme de l'anémie.

Liens d'intérêts Les auteurs déclarent ne pas avoir de liens d'intérêts 


\section{Références}

1. Coppo P, Veyradier A, Loirat C (2012) Microangiopathies thrombotiques : référentiels hémostase/Société française d'hématologie. Hematologie 18:221-32

2. Tadakamalla A, Talluri SK, Besur S (2011) Pseudo-thrombotic thrombocytopenic purpura: a rare presentation of pernicious anemia. N Am J Med Sci 3:472-4

3. Lesesve JF, Salignac S, Bordigoni P, et al (2007) Schistocytes measurement in the laboratory of haematology. Hematologie 13:193-204

4. Noël N, Maigné G, Tertian G, et al (2013) Hemolysis and schistocytosis in the emergency department: consider pseudothrombotic microangiopathy related to vitamin B12 deficiency. QJM $106: 1017-22$
5. Ballas S, Saidi P, Constantino M (1976) Reduced erythrocytic deformability in megaloblastic anemia. Am J Clin Pathol 66:953-7

6. Acharya U, Gau JT, Horvath W, et al (2008) Hemolysis and hyperhomocysteinemia caused by cobalamin deficiency: three case reports and review of the literature. J Hematol Oncol 1:26

7. Loukili N, Andrès E (2008) Vitamine B12 chez l'adulte : du métabolisme aux carences. Ann Endocrinol 64:376-82

8. Loukili N, Noel E, Blaison G, et al (2004) La maladie de Biermer en médecine interne, données actuelles issues d'une étude rétrospective multicentrique de 49 patients. Rev Med Int 25:556-61

9. Andrès E, Kurtz JE, Perrin AE, et al (2001) Oral cobalamin therapy for the treatment of patients with food-cobalamin malabsorption. Am J Med 111:126-9

10. Maes ML, Fixen DR, Linnebur SA (2017) Adverse effects of proton-pump inhibitor use in older adults: a review of the evidence. Ther Adv Drug Saf 8:273-97 